\title{
Editorial
}

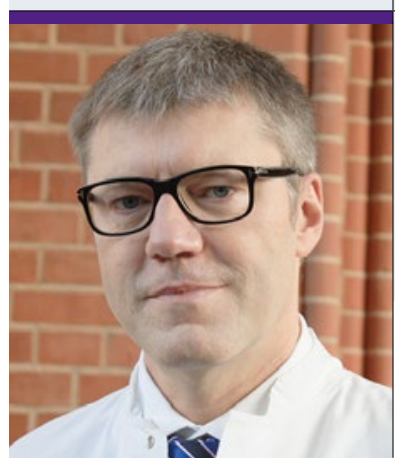

„Warum können sich nicht Urogynäkologen und Hersteller-

firmen an einen Tisch setzen und gemeinsam versuchen

zu definieren, welche aktuellen Erfordernisse vaginale Netze

erfüllen sollten?"

Prof. Dr. med. Ralf Tunn

ist Chefarzt der Klinik für Urogynäkologie und Koordinator des Deutschen Beckenbodenzentrums am St. Hedwig Krankenhaus in Berlin.

\section{Ganz großes Kino}

D er 8. Urogynäkologie-Kongress in Berlin hat den mehr als 500 Teilnehmern wieder eine Plattform für klinische und wissenschaftliche Diskussionen geboten. Das Motto „Ganz großes Kino“ wurde allein schon durch den Tagungsort im Kino KOSMOS erfüllt und durch Filmtrailer in den Pausen sowie eine Podiumsdiskussion über ästhetische Chirurgie (s. a. Beitrag S. 27) mit Modelagentin, Schauspielerin und Dermatologin abgerundet. Typisch für großes Kino ist aber auch das offene Filmende. Die „take home message“ musste jeder für sich selbst finden, meine ist Folgende: In der Hauptsitzung zur Zysto- und Rektozelenkorrektur durch Faszienrekonstruktion fand die lange Tradition dieser Operationstechniken ihre Bestätigung. Die Fascia pubocervicalis wird zur Rekonstruktion der Zystozele benutzt, nach lateral sollte nicht weiter als bis zur Umschlagsfalte präpariert werden, die proximale Begrenzung für die quergestellten Raffnähte ist durch die Blasenpfeiler, die distale durch das Diaphragma urogenitale definiert. Die anatomischen Strukturen zur Rektozelenkorrektur sind unter Einbeziehung ihrer Embryogenese eher unklar definiert. Dem steht gegenüber, dass die Zystozelenkorrektur trotz klar definierter anatomischer Strukturen bis zu $50 \%$ Rezidive hinterlässt und die Rektozelenkorrektur mit ca. $85 \%$ Heilung trotz unklarer Anatomie aufwartet. Eine zusätzliche laterale und vor allem apikale Fixation ist schließlich ein Konzept zur Verbesserung der Heilungsraten nach Zystozelenkorrektur. Alloplastische Netzimplantate erfüllen neben der permanenten Gewebestabilisierung auch diese Bedingungen und scheinen bei Frauen mit Zystozele und Levatorabriss bessere Heilungsraten im Vergleich zur Faszienrekonstruktion zu haben. Das heißt, dass vaginale Netze nicht nur in der Rezidivsituation sondern auch bei hohem Rezidivrisiko indiziert sind, das Gutachten des „scientific committee on emerging and newly identified health risks" (SCENIHR) der Europäischen Union kam 2015 zum gleichen Schluss.

Aber welches Netz dürfen wir nun nehmen? Zur ersten Generation der vaginalen Netze (transobturatorischer Zugangsweg) gibt es mittelfristige Daten, aber die Netze sind teilweise nicht mehr erhältlich und wir nutzen mittlerweile transvaginale Single-Incision-Netze mit geringerer Komplikationsrate. Ein technisch sehr ausgereiftes und wissenschaftlich belegtes Netz hat der Hersteller vom Markt genommen. Solche Szenarien erschweren den Einsatz alloplastischer Netze erheblich, vor allem, weil die wissenschaftlichen Daten mit einem Netz nicht auf ein anderes übertragen werden können. Dieses Dilemma sollte uns zum Umdenken bewegen. Warum können sich nicht Urogynäkologen und Herstellerfirmen an einen Tisch setzen und gemeinsam versuchen zu definieren, welche aktuellen Erfordernisse vaginale Netze erfüllen sollten? Also, einen Rahmen für Netzgewicht, Poren- und Netzgröße sowie anatomischen Fixationspunkte festlegen. Wenn man die angebotenen Netze in diesen Punkten vergleicht, scheint eine Einigung durchaus möglich. Natürlich sollten es Single-Incision-Techniken sein und wissenschaftliche Studien und Herstellerzertifikate vorliegen, zudem muss die Marktbeständigkeit gesichert sein. Ein solches Konsensusmeeting sollte unter Schirmherrschaft der AGUB stattfinden.

Letztlich wollen wir auch in Zukunft die CochraneAnalysen und Leitlinien maßgeblich in unsere klinischen Entscheidungen einbeziehen. Bedenkt man aber, dass bei der Erstellung der aktuellen Descensus-Leitlinie nur 3 von 400 berücksichtigten Publikationen aus Deutschland kamen, so würden unsere täglichen Entscheidungen weitestgehend „fremdgesteuert" sein. Eine traurige Tatsache, bedenkt man, dass Deutschland eine alte Tradition und damit auch Verantwortung bezüglich der vaginalen Operationsschule hat und wir weniger Komplikationen bei der vaginalen Netzchirurgie verzeichnen, zumindest besteht bis heute kein Anstieg der Schlichtungsanfragen. Eigentlich könnte die AGUB ein einheitliches Studienprotokoll für vaginale Netzeinlagen beim Descensus erstellen und alle Firmen sind herzlich eingeladen, als AGUB-Förderer die Finanzierung zu unterstützen, das wäre wirklich großes Kino ...

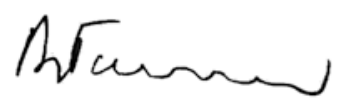

